Originalien

Somnologie $2021 \cdot 25: 205-211$

https://doi.org/10.1007/s11818-021-00293-w

Eingegangen: 28 . September 2020

Angenommen: 2. Januar 2021

Online publiziert: 9 . Februar 2021

(c) Der/die Autor(en) 2021
Michael Schredl' 1 - Maria Schackert ${ }^{2} \cdot$ Gordon Benedikt Feld $^{2} \cdot$ Claudia Schilling $^{2}$

'Schlaflabor, Zentralinstitut für Seelische Gesundheit, Medizinische Fakultät Mannheim, Universität Heidelberg, Mannheim, Deutschland

${ }^{2}$ Zentralinstitut für Seelische Gesundheit, Medizinische Fakultät Mannheim, Universität Heidelberg, Mannheim, Deutschland

\title{
Ein Fragebogen zur Erfassung von schlafbezogenen Metakognitionen: Deutsche Kurzform des MCQ-I
}

\section{Zusatzmaterial online}

Die Online-Version dieses Beitrags (https:// doi.org/10.1007/s11818-021-00293-w) enthält Zusatzmaterial: Fragebogen zu Metakognitionen - Insomnie (MCQ-I 20). Beitrag und Zusatzmaterial stehen Ihnen auf www.springermedizin.de zur Verfügung. Bitte geben Sie dort den Beitragstitel in die Suche ein, das Zusatzmaterial finden Sie beim Beitrag unter „Ergänzende Inhalte“.

\section{Einführung}

Die chronische Insomnie ist gekennzeichnet durch Ein- und/ oder Durchschlafstörungen (mindestens dreimal pro Woche über drei Monate), die zu einer signifikanten Beeinträchtigung der Tagesbefindlichkeit wie Müdigkeit, Konzentrationsproblemen, Leistungseinbußen, vermindertem Antrieb oder Niedergestimmtheit führen [3]. Die Prävalenzrate dieser so definierten chronischen Insomnie ist in der Allgemeinbevölkerung sehr hoch und wird je nach Studie zwischen 9,5 und $13,4 \%$ geschätzt [1]; Frauen sind ca. 1,5 mal so häufig betroffen wie Männer [18].

Den verschiedenen ätiologischen Modellen der Insomnie (Übersicht: [11]) ist gemeinsam, dass prädisponierende, auslösende und aufrechterhaltende Faktoren als wichtig herausgestellt werden. Die aufrechterhaltenen Faktoren werden auch als Teufelskreislauf konzeptualisiert [5], bei dem schlafbehindernde Gedanken, emotionale und physiologische Auswirkungen („Arousal“), negative Konsequenzen des schlechten Schlafs und ungünstige Schlafgewohnheiten sich gegenseitig verstärken. Neben Techniken wie Stimuluskontrolle und Schlafrestriktion stellt das kognitive Umstrukturieren von dysfunktionalen schlafbezogenen Gedanken, z. B. „Acht Stunden Schlaf sind notwendig, um erholt zu sein" einen wichtigen Baustein der kognitiv-verhaltenstherapeutischen Behandlung der Insomnie dar [5].

Auch wenn Entspannungsverfahren, die sowohl auf eine physiologische Entspannung als auch auf einen entspannten Umgang mit Gedanken abzielen, in der Insomnietherapie schon immer eine bedeutende Rolle spielten [5], wird im Zuge der Einführung achtsamkeitsbasierter Verfahren die Rolle von schlafbezogenen Metakognitionen in der Aufrechterhaltung der Insomnie betont, da diese zum Arousal in der Schlafsituation beitragen können [6]. Metakognitionen werden dabei als alle kognitiven Prozesse definiert, die an der Interpretation, dem Monitoring oder der Steuerung von Kognitionen beteiligt sind [16]. „Ich muss schlafen, sonst mache ich bei der Arbeit viele Fehler" wäre eine schlafbezogene Kognition [5], während „Vor dem Einschlafen sollte ich so viele Arten wie möglich ausprobieren, meine Gedanken zu kontrollieren“ eine schlafbezogene Metakognition ist [15]. Für die Therapie bedeutet das, dass weniger auf die inhaltliche Umstrukturierung dysfunktionaler schlafbezogener Kognitionen wertgelegt wird, sondern durch eine Änderung der metakognitiven Überzeugungen ein neuer Umgang mit Kognitionen erreicht wird.

Auch wenn das Einbinden von schlafbezogenen Metakognitionen in die aufrechterhaltenden Faktoren der Insomnie [6] plausibel erscheint, stellt sich die Frage, ob bei Patienten mit Insomnie vermehrt solche schlafbezogenen Metakognitionen auftreten. Um dieser Fragestellung nachzugehen, entwickelten Waine et al. [15] ausgehend von dem Metakognitiven Fragebogen MCQ [17] in Zusammenarbeit mit Insomniepatienten einen Fragebogen, der schlafbezogene Metakognitionen (MCQ-I) erfasst. Der Summenscore der 60 Items der Endfassung zeigte deutlich erhöhte Werte bei der Patientengruppe im Vergleich zu gesunden Schläfern [15]. Zwei Nachfolgestudien [7, 9] konnten diesen Befund replizieren: Insomniepatienten wiesen vermehrte ungünstige schlafbezogene Metakognitionen auf. Auch wenn gute Testkriterien mit hoher Retest-Reliabilität sowie hohe Korrelationen zurSchlafqualität und zum Auftreten allgemeiner Metakognitionen [15] gefunden wurden, stellt sich die Frage, ob für die Erfassung eines einzelnen Konstrukts 60 Items notwendig oder angemessen sind. Zudem zeigte sich, dass einige Items eher schlafbezogene Kognitionen darstellen und nicht schlafbezo- 
Tab. 1 Diagnosen (Mehrfachnennungen möglich) der Gesamtstichprobe und der Substichprobe mit Polsysomnografie (PSG)

\begin{tabular}{|c|c|c|c|c|}
\hline \multirow[t]{2}{*}{ Diagnosen } & \multicolumn{2}{|c|}{$\begin{array}{l}\text { Gesamtstichprobe } \\
(N=512)\end{array}$} & \multicolumn{2}{|c|}{$\begin{array}{l}\text { Substichprobe mit } \\
\text { PSG } \\
(N=348)\end{array}$} \\
\hline & Häufigkeit & Prozent & Häufigkeit & Prozent \\
\hline Insomnie & 111 & 21,68 & 168 & 51,59 \\
\hline Insomnie (Verdacht) & 144 & 28,13 & 0 & 0,00 \\
\hline $\begin{array}{l}\text { Restless-Legs-Syndrom (RLS)/Periodische Bein- } \\
\text { bewegungen im Schlaf (PLMS) }\end{array}$ & 65 & 12,70 & 117 & 33,72 \\
\hline $\begin{array}{l}\text { Restless-Legs-Syndrom (RLS)/Periodische Bein- } \\
\text { bewegungen im Schlaf (PLMS) (Verdacht) }\end{array}$ & 87 & 16,99 & $5^{\mathrm{a}}$ & 1,44 \\
\hline Hypersomnie/Narkolepsie & 12 & 2,34 & 16 & 4,61 \\
\hline Hypersomnie/Narkolepsie (Verdacht) & 36 & 7,03 & 1 & 0,29 \\
\hline Alptraumstörung & 50 & 9,77 & 33 & 9,51 \\
\hline $\begin{array}{l}\text { REM-Parasomnien (gesicherte und Verdachts- } \\
\text { diagnosen) }\end{array}$ & 35 & 6,84 & 23 & 6,63 \\
\hline NREM-Parasomnien & 29 & 5,66 & 27 & 7,78 \\
\hline NREM-Parasomnien (Verdacht) & 11 & 2,15 & 3 & 0,86 \\
\hline $\begin{array}{l}\text { Schlafbezogene Atemregulationsstörung } \\
\text { (SBAS) }\end{array}$ & 49 & 9,57 & 73 & 21,04 \\
\hline $\begin{array}{l}\text { Schlafbezogene Atemregulationsstörung } \\
\text { (SBAS) (Verdacht) }\end{array}$ & 42 & 8,20 & $7^{a}$ & 2,02 \\
\hline Störung des Schlaf-Wach-Rhythmus & 31 & 6,05 & 21 & 6,05 \\
\hline Depressive Störung & 116 & 22,66 & 98 & 28,24 \\
\hline Andere psychische Störungen & 50 & 9,77 & 38 & 10,95 \\
\hline Sonstige Schlafstörungen & 37 & 7,23 & 65 & 18,73 \\
\hline $\begin{array}{l}\text { Somatische Erkrankungen, die mit Schlafstö- } \\
\text { rung in direktem Zusammenhang stehen }\end{array}$ & 15 & 2,93 & 6 & 1,73 \\
\hline Ohne Diagnose & 4 & 0,78 & 3 & 0,86 \\
\hline
\end{tabular}

gene Metakognitionen, z.B. „Wenn ich wach im Bett liege, bedeutet das, dass ich am nächsten Tag nicht funktionieren werde“ oder „Das kleinste Geräusch bedeutet, dass meine Chance zu schlafen gefährdet sein wird“ [15]. Das heißt, dass einige Items nicht auf den Umgang mit Gedanken in der Schlafsituation abzielen, sondern die typischen dysfunktionalen Kognitionen erfassen, die bei Insomniepatienten auftreten können.

Das Ziel der vorliegenden Arbeit war es, eine kürzere Version des MCQ-I in deutscher Sprache zu erstellen, die Items enthält, die sich klar auf schlafbezogene Metakognitionen beziehen. Dabei sollten sowohl eine hohe interne Konsistenz als auch eine hohe Retest-Reliabilität erreicht werden. Um die Validität der Skala nachzuweisen, wurde geprüft, ob dysfunktionale schlafbezogene Metakognitionen bei Patienten mit einer Insomnie- diagnose häufiger auftreten als bei Patienten mit anderen Schlafstörungen.

\section{Methode}

\section{Patienten}

Insgesamt konnten 512 Patienten (264 Frauen, 248 Männer) in die Auswertung eingeschlossen werden. Der Altersmittelwert lag bei 45,04 \pm 16,70 Jahren. Ein Teil der Patienten $(N=347)$ wurde nach dem Ambulanztermin auch polysomnografisch im Schlaflabor über zwei Nächte untersucht. Diese Substichprobe bestand aus 179 Frauen und 168 Männern. Der Altersmittelwert lag hier bei 44,52 $\pm 16,22$ Jahren. Die Verteilungen der Diagnosen (Mehrfachdiagnosen waren möglich) sind in $\bullet$ Tab. 1 dargestellt.

Für eine Subgruppe von $N=19$ Insomniepatienten (16 Frauen, 3 Männer) mit dem Altersmittelwert von 46,84 $\pm 12,41$
Jahren erfolgte eine zweite Messung, da sie den MCQ-I (deutsche Fassung) vor Beginn der Gruppentherapie (kognitive Therapie der Insomnie) zum zweiten Mal ausfüllten. In dieser Gruppe traten zusätzlich zur Insomnie folgende schlafbezogene Komorbiditäten auf: RestlessLegs-Syndrom $(N=2)$, schlafbezogene Atemregulationsstörung $(N=2)$, periodische Beinbewegungen im Schlaf $(N=1)$, eine isolierte Schlafparalyse $(N=1)$ und eine leichte depressive Symptomatik $(N=1)$. Das mittlere RetestIntervall lag bei $147,42 \pm 68,11$ Tagen.

\section{Fragebogen zu schlafbezogenen Metakognitionen (MCQ-I)}

Ausgehend vom Metakognitiven Fragenbogen (MCQ-30; [17]) wurde von Waine et al. [15] der Fragebogen zu schlagebezogenen Metakognitionen in Zusammenarbeit mit Insomniepatienten entwickelt. Er besteht aus 60 Aussagen mit vier Antwortmöglichkeiten ( $1=$ Stimme überhaupt nicht $\mathrm{zu}, 2$ = Stimme leicht $\mathrm{zu}$, $3=$ Stimme mäßig zu und $4=$ Stimme voll und ganz zu). Beispielitems sind: „Vor dem Einschlafen sollte ich stressbehaftete Gedanken durch weniger stressbehaftete ersetzen“ oder „Vor dem Einschlafen sollte ich an Dinge denken, die keine Bedeutung für mich haben“. Der Gesamtscore wird als Summenwert über alle 60 Items berechnet; höhere Werte weisen in Richtung problematischer Metakognitionen. Wenn mehr als 5 Items nicht ausgefüllt wurden, wurde der Gesamtwert nicht berechnet (missing). Die Testautoren geben eine Retest-Reliabilität (4 Wochen) von $r=0,82$ für die Gesamtstichprobe und $r=0,93$ für die Gruppe der Insomniepatienten an [15]. Aufgrund der geringen Stichprobengröße $(N=71)$ wurde keine interne Konsistenz (Crohnbachs Alpha) berechnet. Der MCQ-IGesamtscore korreliert mit $\mathrm{r}=0,59 \mathrm{mit}$ dem Gesamtscore des Pittsburgh Sleep Quality Index (PSQI) und mit $r=0,69$ mit dem Metakognitiven Fragebogen (MCQ-30) [15]. Zudem zeigte sich ein deutlicher Unterschied (große Effektstärke) zwischen den Insomniepatienten und den Schlafgesunden. Dies wurde in zwei weiteren Studien [7, 9] bestätigt; auch wiesen Insomniepatienten höhere 
MCQ-I-Werte auf als Personen, die eine reduzierte Schlafqualität in Verbindung mit Schnarchen aufwiesen [9].

\section{Versuchsablauf}

Vor dem Gesprächstermin in der Schlafambulanz (Zentralinstitut für Seelische Gesundheit) werden den Patienten Fragebogen zugeschickt, z. B. Epworth Sleepiness Scale (ESS), und auch der MCQ-I in der deutschen Übersetzung (siehe unten). Diese Fragebogen sollen ausgefüllt zum Termin mitgebracht werden. Die Ethikkommission II der Medizinischen Fakultät Mannheim/Universität Heidelberg hatte nach eingehender Prüfung keine ethischen und/oder berufsrechtlichen Einwände gegen die Auswertung dieser klinischen Daten. Da die Fragebogen Teil der klinischen Routine sind, wurden sie von allen Patienten ausgefüllt; nur in vier Fällen wurde der Fragebogen aufgrund von Sprachproblemen leer abgegeben. Allerdings wurden einige Fragebogen unvollständig ausgefüllt (dies betraf vor allem die Rückseiten des doppelseitig ausgedruckten Fragebogens), sodass es zu einer Reduktion der auswertbaren Fragebogen kam. Nach dem Ambulanzgespräch (geführt durch in Schlafmedizin erfahrene Ärztinnen) wurden die Diagnosen bzw. Verdachtsdiagnosen gestellt. Ein Teil der Patienten wurde dann polysomnografisch im Schlaflabor (zwei Nächte) untersucht, sodass die abschließende Diagnose diese Befunde mit einbeziehen konnte. Als zertifiziertes Schlaflabor der Deutschen Gesellschaft für Schlafforschung und Schlafmedizin (DGSM) folgten die Polysomnografie (PSG) und deren Auswertung den Richtlinien der American Academy of Sleep Medicine [2].

Für die Studie zur Entwicklung der Kurzform des MCQ-I wurden die Diagnosen der Patienten in verschiedenen Gruppen zusammengefasst. Dabei ist zu beachten, dass Patienten mehrere Diagnosen aufweisen konnten. Zunächst wurde die Diagnose der Insomnie bzw. die Verdachtsdiagnose der Insomnie gesondert behandelt, da hier die Validität des MCQ-I geprüft wurde. RestlessLegs-Syndrom und periodische Bein-

Somnologie 2021 ·25:205-211 https://doi.org/10.1007/s11818-021-00293-W

(c) Der/die Autor(en) 2021

M. Schredl · M. Schackert · G. B. Feld · C. Schilling

Ein Fragebogen zur Erfassung von schlafbezogenen Metakognitionen: Deutsche Kurzform des MCQ-I

\section{Zusammenfassung}

Hintergrund und Fragestellung. Schlafbezogene Kognitionen, aber auch schlafbezogene Metakognitionen (Umgang mit Gedanken) spielen in der Ätiologie der Insomnie, vor allem bei der Aufrechterhaltung, eine Rolle. Bisher liegt nur ein englischsprachiger Fragebogen (MCQ-I) mit 60 Items zur Erfassung schlafbezogener Metakognitionen vor. Ziel dieser Arbeit war das Zusammenstellen einer kürzeren, deutschen Version, die sowohl gute Reliabilität als auch Validität aufweist.

Material und Methoden. Insgesamt nahmen 512 Patienten mit unterschiedlichen Schlafstörungen (264 Frauen, 248 Männer; mittleres Alter: 45,04 $\pm 16,70$ Jahre) an der Erhebung teil. Der MCQ-I wurde vor dem Erstgespräch ausgefüllt mitgebracht. Ergebnisse. Die 20-Item-Version wies eine hohe interne Konsistenz $(r=0,906)$ und hohe Retest-Reliabilität $(r=0,916)$ auf. Patienten mit der Diagnose einer Insomnie zeigten einen signifikant höheren Summenwert im MCQ-I 20 als Patienten mit anderen Schlafstörungen. Interessanterweise waren auch die Diagnose einer depressiven Erkrankung und das Auftreten einer Alptraumstörung mit vermehrten schlafbezogenen Metakognitionen verbunden.

Schlussfolgerung. Schlafbezogene Metakognitionen scheinen in der InsomnieÄtiologie eine Rolle zu spielen. Interessant wäre es zu überprüfen, ob eine rein metakognitive Therapie die Symptomatik bei Insomniepatienten in ähnlicher Weise wie die klassische kognitive Verhaltenstherapie der Insomnie verbessern kann.

\section{Schlüsselwörter}

Ein- und Durchschlafstörungen · Befragungen und Fragebögen · Depression · Insomnie · Kognitive Verhaltenstherapie

\section{A questionnaire for measuring sleep-related metacognitions: a German short version of the MCQ-I}

Abstract

Background and objectives. Sleeprelated cognitions, but also sleep-related metacognitions (dealing with cognitions), play a role in insomnia etiology, especially in maintaining insomnia. So far, an English version of a questionnaire measuring sleeprelated metacognitions with 60 items is available. The aim of the present study was the construction of a shorter German version showing satisfactory reliability and validity. Materials and methods. Overall, 512 patients with varying sleep disorders (264 women, 248 men; mean age: $45.04 \pm 16.70$ years) participated. The MCQ-I was completed prior to the consultation in the sleep center. Results. The 20 -item version showed high internal consistency $(r=0.906)$ and high retest reliability $(r=0.916)$. Patients with insomnia diagnosis had higher MCQ-I 20 sum scores compared to patients with other sleep disorders. Interestingly, patients with diagnoses of depressive disorder or nightmare disorder also reported higher values of sleeprelated metacognitions.

Conclusion. Sleep-related metacognitions seem to play a role in insomnia etiology. It would be very interesting to test whether metacognitive therapy can be as effective as cognitive-behavioral therapy for insomnia in alleviating insomnia symptomatology.

Keywords

Sleep initiation and maintenance disorders . Surveys and questionnaires - Depression . Insomnia · Cognitive behavioral therapy bewegungen im Schlaf wurden zusammengefasst in eine Gruppe mit gesicherten Diagnosen und eine Gruppe mit Verdachtsdiagnosen (Ambulanzgespräch) bzw. Grenzbefunden (PSGSubstichprobe). Aufgrund der kleinen Fallzahlen wurden Narkolepsie und Hy- persomnie zusammengefasst. In der Gruppe der NREM-Parasomnien sind Pavor nocturnus, Schlafwandeln und Schlaftrunkenheit enthalten. Die schlafbezogenen Atemregulationsstörungen enthalten die obstruktive Schlafapnoe und das obstruktive Schnarchen. Die 
Tab. 20 Items mit schlafbezogenen Metakognitione

\begin{tabular}{|c|c|c|c|c|}
\hline Item & $\begin{array}{l}\text { Initiale } \\
\text { Ratings }^{\text {a }}\end{array}$ & $M \pm S D$ & $N$ & $\begin{array}{l}\text { Trenn- } \\
\text { schärfe }\end{array}$ \\
\hline $\begin{array}{l}\text { 1. Wenn ich gedanklich im Bett nicht zur Ruhe kom- } \\
\text { me, bedeutet das, dass ich keine Kontrolle über } \\
\text { meine Gedanken habe }\end{array}$ & 3 & $2,28 \pm 1,13$ & 456 & 0,623 \\
\hline $\begin{array}{l}\text { 2. Vor dem Einschlafen sollte ich meine Gedanken } \\
\text { genau untersuchen }\end{array}$ & 2 & $1,69 \pm 0,88$ & 455 & 0,587 \\
\hline $\begin{array}{l}\text { 3. Wenn ich im Bett nachdenke, bedeutet das, dass } \\
\text { ich gestresst bin }\end{array}$ & 2 & $2,24 \pm 1,06$ & 459 & 0,590 \\
\hline $\begin{array}{l}\text { 4. Vor dem Einschlafen sollte ich mich auf Körper- } \\
\text { empfindungen, die mich am Schlafen hindern könn- } \\
\text { ten, überprüfen }\end{array}$ & 2 & $2,07 \pm 1,03$ & 454 & 0,491 \\
\hline $\begin{array}{l}\text { 5. Vor dem Einschlafen sollte ich stressbehaftete } \\
\text { Gedanken durch weniger stressbehaftete ersetzen }\end{array}$ & 3 & $2,65 \pm 1,06$ & 457 & 0,564 \\
\hline $\begin{array}{l}\text { 6. Wenn ich gedanklich im Bett nicht zur Ruhe kom- } \\
\text { me, wird mir das schaden }\end{array}$ & 3 & $2,55 \pm 1,13$ & 461 & 0,675 \\
\hline $\begin{array}{l}\text { 7. Während ich im Bett bin, sollte ich überprüfen, wie } \\
\text { nah ich am Einschlafen bin }\end{array}$ & 2 & $1,57 \pm 0,86$ & 459 & 0,575 \\
\hline $\begin{array}{l}\text { 8. Wenn ich wach im Bett liege, sollte ich mich auf } \\
\text { Erinnerungen an frühere Nächte verlassen, um her- } \\
\text { auszufinden, warum }\end{array}$ & 2 & $1,40 \pm 0,75$ & 457 & 0,537 \\
\hline $\begin{array}{l}\text { 9. Im Bett nachzudenken bedeutet, dass ich nicht } \\
\text { einschlafen werde }\end{array}$ & 3 & $2,36 \pm 1,11$ & 457 & 0,687 \\
\hline $\begin{array}{l}\text { 10. Vor dem Einschlafen sollte ich so viele Arten } \\
\text { wie möglich ausprobieren, meine Gedanken zu } \\
\text { kontrollieren }\end{array}$ & 2 & $1,79 \pm 0,96$ & 458 & 0,687 \\
\hline $\begin{array}{l}\text { 11. Vor dem Einschlafen sollte ich Zeit darauf ver- } \\
\text { wenden, über die Zukunft nachzudenken }\end{array}$ & 2 & $1,48 \pm 0,86$ & 458 & 0,536 \\
\hline $\begin{array}{l}\text { 12. Wenn ich im Bett nachdenke, bedeutet das, dass } \\
\text { ich am nächsten Tag nicht gut leistungsfähig sein } \\
\text { werde }\end{array}$ & 1 & $2,30 \pm 1,09$ & 460 & 0,628 \\
\hline $\begin{array}{l}\text { 13. Wenn ich gedanklich im Bett nicht zur Ruhe } \\
\text { komme, bedeutet das, dass ich nicht normal bin }\end{array}$ & 3 & $1,45 \pm 0,84$ & 460 & 0,495 \\
\hline $\begin{array}{l}\text { 14. Wenn ich gedanklich im Bett nicht zur Ruhe } \\
\text { komme, bedeutet das, dass ich über die falschen } \\
\text { Dinge nachdenke }\end{array}$ & 3 & $2,11 \pm 1,06$ & 460 & 0,639 \\
\hline $\begin{array}{l}\text { 15. Bevor ich einschlafe, sollte ich überwachen, wie } \\
\text { entspannt mein Körper ist }\end{array}$ & 2 & $1,76 \pm 0,92$ & 457 & 0,611 \\
\hline $\begin{array}{l}\text { 16. Vor dem Einschlafen sollte ich an Dinge denken, } \\
\text { die keine Bedeutung für mich haben }\end{array}$ & 2 & $1,82 \pm 1,00$ & 458 & 0,573 \\
\hline $\begin{array}{l}\text { 17. Vor dem Einschlafen sollte ich versuchen, meine } \\
\text { Gedanken abzuschalten }\end{array}$ & 2 & $2,53 \pm 1,11$ & 459 & 0,673 \\
\hline $\begin{array}{l}\text { 18. Wenn ich im Bett nachdenke, bedeutet das, dass } \\
\text { ich den nächsten Tag nicht bewältigen werde }\end{array}$ & 3 & $1,81 \pm 0,97$ & 457 & 0,626 \\
\hline $\begin{array}{l}\text { 19. Bevor ich einschlafe, muss ich die Dinge in mei- } \\
\text { nem Kopf sortiert bekommen }\end{array}$ & 2 & $1,96 \pm 0,98$ & 460 & 0,656 \\
\hline $\begin{array}{l}\text { 20. Vor dem Einschlafen sollte ich Zeit darauf ver- } \\
\text { wenden, über kurz zurückliegende Ereignisse nach- } \\
\text { zudenken }\end{array}$ & 1 & $1,72 \pm 0,88$ & 459 & 0,520 \\
\hline
\end{tabular}

Rhythmusstörungen waren ausschließlich verzögerte Schlafphasensyndrome. Innerhalb der depressiven Erkrankungen waren sowohl majore depressive Störungen als auch Dysthymie gruppiert. In der Restgruppe der psychischen Stö- rungen wurden Angststörungen, posttraumatische Belastungsstörung, emotional-instabile Persönlichkeitsstörung, Zwangsstörungen, Essstörungen usw. zusammengefasst. Die sonstigen Schlafstörungen bestanden vorwiegend aus dem Schlafmangelsyndrom und schlafbezogenen rhythmischen Bewegungsstörungen. Wenn die somatische Diagnose für die Schlafstörung eine wesentliche Rolle spielte, wurde diese kodiert, z.B. Schmerzsyndrome, Tinnitus, Epilepsie.

Die Übersetzung und Verkürzung des MCQ-I-Fragebogen wurde wie folgt durchgeführt. Claudia Schilling übersetzte den Fragebogen von Waine et al. [15] ins Deutsche. Die Rückübersetzung wurde von Gordon Benedikt Feld durchgeführt; und diese Rückübersetzung wurde von Niall Broomfield, einem der Testautoren des MCQ-I, mit dem Original verglichen. Es ergaben sich minimale Veränderungen, die jedoch aufgrund eines technischen Fehlers nicht für diese Stichprobe umgesetzt wurden, z. B. wurden statt der Kategorien „Stimme überhaupt nicht zu“, „Stimme leicht zu“, „Stimme mäßig zu“ und „Stimme voll und ganz zu“ (korrigiere Fassung) die Kategorien „Trifft nicht zu“, „Trifft etwas zu“, „Trifft mäßig zu“ und „Trifft sehr stark zu“ der Vorfassung verwendet. Drei deutschsprachige, zertifizierte MCT-Therapeutinnen (Level 1 des Metakognitive Therapy Institutes von Dr. Adrian Wells und Dr. Hans Nordahl) beurteilten unabhängig voneinander, ob die Items des MCQ-I (deutsche Version) den Grundprinzipien von Metakognitionen entsprachen und Metakognitionen in gut verständlicher und eindeutiger Weise abfragten. Eine der Beurteilerinnen hatte auch die Zusatzbezeichnung Schlafmedizin. Nach diesem Schritt erfolgte eine Beratung, in der die drei Beurteilerinnen sich auf die 20 Items einigten, die auf eindeutige Weise schlafbezogene Metakognitionen erfassen.

Die statistischen Analysen wurden mit SAS 9.4 für Windows (Cary, NC, USA) durchgeführt. Zunächst wurden die Testgütekriterien der 20-Item-Version (Trennschärfen, interne Konsistenz, Retest-Reliabilität) berechnet. Trennschärfe bezeichnet hierbei die Korrelation eines Einzelitems mit dem Summenwert aller Items; hohe Trennschärfen sind ein Indiz, dass alle Items das gleiche Konstrukt (hier: Ausprägung schlafbezogener Metakognitionen) messen. In ähnlicher Weise gibt die interne Konsistenz, auch Crohnbachs Alpha genannt, an, wie 


\begin{tabular}{|c|c|c|c|c|c|c|}
\hline \multirow[t]{2}{*}{ Variablen } & \multicolumn{3}{|c|}{$\begin{array}{l}\text { Gesamtstichprobe } \\
(N=460)\end{array}$} & \multicolumn{3}{|c|}{$\begin{array}{l}\text { Subgruppe mit PSG } \\
(N=314)\end{array}$} \\
\hline & SE & $\mathbf{t}$ & $p$ & SE & $\mathbf{t}$ & $p$ \\
\hline Alter & $-0,0644$ & $-1,2$ & 0,2271 & $-0,0464$ & $-0,7$ & 0,4950 \\
\hline Geschlecht $(1=w, 0=m)$ & 0,0626 & 1,3 & 0,1842 & 0,0351 & 0,6 & 0,5556 \\
\hline Insomnie & 0,1544 & 3,0 & $0,0018^{\mathrm{a}}$ & 0,1142 & 1,8 & $0,0370^{\mathrm{a}}$ \\
\hline Insomnie (Verdacht) & 0,0935 & 1,8 & $0,0386^{\mathrm{a}}$ & - & - & - \\
\hline RLS/PLMS & 0,0253 & 0,5 & 0,5948 & $-0,0721$ & $-1,2$ & 0,2411 \\
\hline RLS/PLMS (Verdacht) ${ }^{\mathrm{b}}$ & 0,0077 & 0,2 & 0,8715 & $-0,0322$ & $-0,6$ & 0,5348 \\
\hline Hypersomnie/Narkolepsie & $-0,0590$ & $-1,3$ & 0,2048 & $-0,1313$ & $-2,2$ & 0,0283 \\
\hline Hypersomnie/Narkolepsie (Verdacht) & $-0,1220$ & $-2,4$ & 0,0178 & 0,0639 & 1,2 & 0,2526 \\
\hline Alptraumstörung & 0,0950 & 2,0 & 0,0433 & 0,0899 & 1,6 & 0,1136 \\
\hline $\begin{array}{l}\text { REM-Parasomnien (Verdachtsdiagnosen und } \\
\text { gesicherte Diagnose) }\end{array}$ & 0,0115 & 0,2 & 0,8125 & $-0,0038$ & $-0,1$ & 0,9500 \\
\hline NREM-Parasomnien & 0,0497 & 1,0 & 0,3266 & 0,0671 & 1,1 & 0,2693 \\
\hline NREM-Parasomnien (Verdacht) & 0,0053 & 0,1 & 0,9082 & 0,0216 & 0,4 & 0,6954 \\
\hline SBAS & 0,0323 & 0,7 & 0,4953 & 0,0767 & 1,2 & 0,2150 \\
\hline SBAS (Verdacht) ${ }^{b}$ & 0,0771 & 1,7 & 0,0970 & $-0,0270$ & $-0,5$ & 0,6329 \\
\hline Störung des Schlaf-Wach-Rhythmus & $-0,0117$ & $-0,2$ & 0,8083 & $-0,0307$ & $-0,5$ & 0,5983 \\
\hline Depressive Störung & 0,1757 & 3,8 & 0,0002 & 0,2000 & 3,3 & 0,0010 \\
\hline Andere psychische Störungen & 0,0884 & 1,9 & 0,0623 & 0,0397 & 0,7 & 0,4879 \\
\hline Sonstige Schlafstörungen & 0,0471 & 1,0 & 0,3281 & $-0,0691$ & $-1,2$ & 0,2316 \\
\hline $\begin{array}{l}\text { Somatische Erkrankungen, die mit Schlaf- } \\
\text { störung in direktem Zusammenhang stehen }\end{array}$ & $-0,0021$ & $-0,1$ & 0,9639 & $-0,0026$ & $-0,1$ & 0,9628 \\
\hline Ohne Diagnose & $-0,0364$ & $-0,8$ & 0,4262 & 0,0036 & 0,1 & 0,9484 \\
\hline \multicolumn{7}{|c|}{ 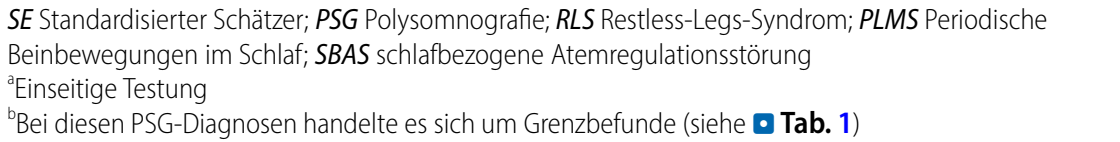 } \\
\hline
\end{tabular}

stark alle Einzelitems miteinander korrelieren. Werte höher als 0,80 werden als gut bezeichnet [12]. Auch bei der Retest-Reliabilität werden bei stabilen Merkmalen Korrelationen von $r=0,80$ oder höher als gut angesehen, d.h., die Versuchspersonen geben bei einer wiederholten Messung ähnliche Werte an, das Konstrukt (hier: Ausprägung schlafbezogener Metakognitionen) ist zeitlich stabil. Bei der 20-Item-Version wurden keine Summenscores berechnet, wenn mehr als zwei Items nicht ausgefüllt wurden. In zwei Regressionsanalysen wurde der Einfluss der Diagnosen auf den Summenscore des MCQ-I 20 überprüft, dabei wurden die Analysen für Alter und Geschlecht kontrolliert. Der standardisierte Schätzer wird auch als Regressionsgewicht bezeichnet und gibt die Größe des Zusammenhangs dieser Variable auf den Prädiktor an, wobei alle Assoziationen von anderen Variablen statistisch herausgerechnet sind. stressbehaftete ersetzen"). Alle 20 Items zeigten eine hohe Trennschärfe zum Summenscore, der über die 20 Items gebildet wurde ( $\bullet$ Tab. 2). Der Mittelwert des Summenscores lag bei 39,56 $\pm 11,89$ $(N=461)$. Die interne Konsistenz betrug $r_{t t}=0,906$. Der Mittelwert über alle 60 Items lag bei 123,15 $\pm 34,26(N=458)$. Für die Vollversion lag die interne Konsistenz bei $r_{t t}=0,961$. Die Korrelation zwischen dem Summenwert der 20Item-Version und der 60-Item-Version war sehr hoch $(r=0,953, p<0,0001$, $N=458)$.

Innerhalb des Retest-Intervalls von durchschnittlich ca. 150 Tagen nahm der MCQ-I-20-Summenwert leicht ab ( $\mathrm{T}_{1}: 40,61 \pm 10,96$ vs. $\mathrm{T}_{2}: 38,32 \pm 11,53$, $\mathrm{t}=-2,2, p=0,0450, N=19)$. Die Korrelation der zwei Testwerte war sehr hoch: $r=0,916(p<0,0001)$. Auch für den Summenscore der Vollversion (60 Items) lag die Retest-Reliabilität sehr hoch: $r=0,919$ $(p<0,0001)$.

Der Einfluss der verschiedenen Störungsbilder auf den Summenwert der 20-Item-Version ist in Dab. 3 dargestellt. Wie zu erwarten zeigte sich für die Gesamtgruppe ein signifikanter Einfluss der Insomniediagnose, auch Patienten mit einem Verdacht einer Insomnie wiesen höhere Werte auf. Interessanterweise ging die Diagnose einer komorbiden depressiven Störung auch mit erhöhten MCQ-I-20-Summenscores einher. Des Weiteren zeigten sich auch erhöhte Werte bei Patienten mit einer Alptraumstörung, während bei der Gruppe mit Verdacht auf eine Hypersomnie oder Narkolepsie die MCQ-I-20-Werte niedriger im Vergleich zur Gesamtgruppe der Patienten waren (negative standardisierte Schätzer in der Regressionsanalyse). Für die Subgruppe der Patienten, die zusätzlich zum Ambulanzgespräch auch polysomnografisch untersucht wurden, bestätigte sich der Einfluss der Insomniediagnose, die mit erhöhten Werten bzgl. schlafbezogener Metakognitionen einhergingen (siehe $\bullet$ Tab.3).

Ebenso zeigte sich wieder der Effekt der komorbiden depressiven Störung. Patienten mit Hypersomnie oder Narkolepsie wiesen niedrigere MCQ-I-20-Werte auf. Alter und Geschlecht hatten (unter Kontrolle der gestellten Diagnosen) kei- 
nen Einfluss auf die Ausprägung schlafbezogener Metakognitionen.

Für den Vergleich mit den bisherigen MCQ-I-Studien wurden die Mittelwerte der 60-Item-Version für Patienten mit der Diagnose einer Insomnie $(131,93 \pm 35,43 ; \quad N=97)$, einer Verdachtsdiagnose einer Insomnie $(127,87 \pm 31,06 ; N=130)$ und der Restgruppe $\quad(116,40 \pm 34,06 ; \quad N=231)$ berechnet.

\section{Diskussion}

Die vorliegende Studie zeigt, dass eine deutsche 20-Item-Version des Fragebogens zur Erfassung schlafbezogener Metakognitionen sowohl eine gute Reliabilität (interne Konsistenz, Retest-Reliabilität) aufweist als auch eine gute Validität, d.h., Patienten mit der Diagnose einer Insomnie weisen höhere Werte auf als Patienten mit anderen Schlafstörungen. Ebenfalls steht der Befund, dass Patienten mit einer Depressionsdiagnose höhere Werte aufweisen, in Einklang mit der Theorie der Metakognitionen, da Grübeln, ein dysfunktionaler Umgang mit Gedanken, ein Kernsymptom depressiver Störungen darstellt.

Um die Ergebnisse der Untersuchung einordnen zu können, ist eine methodische Betrachtungen angebracht. Der erste Punkt bezieht sich auf die fehlende Gruppe gesunder Kontrollen. In den Studien der Arbeitsgruppe um Palagini [7-9] variieren die MCQ-I-Mittelwerte bei den gesunden Schläfern von 61 bis 66; bei einem Range von 60 bis 240 der 60 -ItemVersion bedeutet das, dass hier so gut wie keine dysfunktionalen schlafbezogenen Metakognitionen auftraten. In der Untersuchung von Waine et al. [15] lag in der Gruppe von normalen Schläfern der Mittelwert bei 90, d.h., hier wurden auch dysfunktionale schlafbezogene Metakognitionen gefunden, jedoch deutlich geringer ausgeprägt als bei Insomniepatienten, die Mittelwerte von 122 bis 130 aufwiesen [7-9, 15]. Hier fügen sich unsere aktuellen Werte bei Insomnie (Mittelwert: 131) und bei Personen mit Verdacht auf Insomnie (Mittelwert: 128) sehr gut ein. Allerdings zeigte sich auch, dass sich für die Patienten ohne Insomnie bzw. Insomnieverdacht auch deutlich höhere
Werte (Mittelwert: 116) abzeichnen als bei gesunden Schläfern. Möchte man den MCQ-I 20 in der Differenzialdiagnostik der Insomnie einsetzen, wäre es sinnvoll, Cut-offs zu anderen Schlafstörungen wie z. B. dem Restless-Legs-Syndrom zu berechnen. Da hier die Überlappungen höher sind als zu gesunden Kontrollen, sind größere Stichproben - im Vergleich zur vorliegenden Studie - notwendig. Ein methodisch wichtiger Gesichtspunkt ist, dass die MCQ-I-Werte der Patienten nicht in die diagnostische Entscheidung eingegangen sind, sondern erst zu einem späteren Zeitpunkt für die durchgeführte Analyse ausgewertet wurden. In dieser Erhebung, deren Hauptziel die Entwicklung einer kürzeren Fassung des MCQ-I in deutscher Sprache war, wurden Daten $\mathrm{zu}$ somatischer Komorbidität und Medikamenteneinnahme nicht miteinbezogen. Auch wenn ein eindeutiger Effekt der Insomniediagnose auf die Ausprägung dysfunktionaler schlafbezogener Metakognitionen in der Gesamtgruppe nachweisbar war, wäre es interessant, an einer größeren Stichprobe von Patienten mit Insomnie zu untersuchen, ob die Einnahme von Medikamenten wie Benzodiazepinrezeptoragonisten oder sedierenden Antidepressiva [14] einen Einfluss auf metakognitive Prozesse in dieser Patientengruppe hat.

Ein zweiter methodischer Punkt ist die relativ hohe Zahl von Missings (458 Summenwerte von 512 Patienten konnten berechnet werden). Dies lag zum einen an Sprachproblemen, aber auch daran, dass der vierseitige Test (Vorder- und Rückseite) von einer ganzen Reihe Patienten nur unvollständig ausgefüllt wurde. Es ist zu anzunehmen, dass diese Probleme bei der 20-Item-Version (eine Seite; siehe Fragebogen im elektronischen Zusatzmaterial), weniger häufig auftreten. Durch ein Versehen erhielt die aktuelle Stichprobe nicht die abschließende Endversion der deutschen Übersetzung des MCQ-I; allerdings ist zu vermuten, dass die minimalen sprachlichen Veränderungen keinen Einfluss aufdie Reliabilitätskennwerte bzw. die Ergebnisse der Regressionsanalysen haben. Es ist eine Nachfolgeerhebung mit der Endfassung (siehe elektronisches Zusatzmaterial) geplant. Ein Vorteil der vorliegenden Erhebung ist, dass die Patienten den metakognitiven Fragebogen im klinischen Setting und vor dem Ambulanzgespräch - also vor dem Kontakt mit einem Schlafspezialisten - ausgefüllt haben. Die Retest-Daten lassen vermuten, dass ein ausführliches diagnostisches Gespräch schon einen positiven Einfluss auf die schlafbezogenen Metakognitionen haben kann.

Das verstärkte Auftreten von schlafbezogenen Metakognitionen bei Insomniepatienten, das in den früheren Studien $[7,9,15]$ gezeigt wurde, konnte repliziert werden. In Bezug auf die Gesamtgruppe der Patienten mit Schlafstörungen waren diese erhöhten Werte besonders in der Gruppe mit Insomniediagnose zu finden. Dieser Befund unterstützt das metakognitive Modell der Insomnie-Ätiologie, das von Ong et al. [6] formuliert wurde. Interessanterweise passt die enge Korrelation zwischen dem Gesamtwert der 60Item-Version, die viele Items mit schlafbezogenen Kognitionen enthält, und dem Summenwert der 20-Item-Version, die auf die Messung schlafbezogener Metakognitionen abzielt, auch in dieses zweistufige Modell, da das schlafbezogene Arousal im ersten Schritt von den schlafbezogenen Kognitionen getrieben wird, jedoch die schlafbezogenen Metakognitionen im zweiten Schritt das Arousal noch weiter ansteigen lassen [6]. Weitere Studien sind notwendig, um diese beiden Konzepte stärker zu differenzieren.

Theoriekonform ist auch das erhöhte Auftreten von schlafbezogenen Metakognitionen bei Personen mit einer Depressionsdiagnose. Im metakognitiven Modell der Depression [16] spielt das Grübeln eine bedeutende Rolle, weil hier eine starke Unkontrollierbarkeitsüberzeugung und teilweise auch eine starke Metakognition zum Nutzen des Grübelns sowie ein verringertes Metabewusstsein für den Prozess des Grübelns vorliegen. Auch die Wirksamkeit von kognitiver Verhaltenstherapie der Insomnie bei Patienten mit einer Depression [4] könnte darauf hinweisen, dass depressive Patienten nicht nur eine allgemein erhöhte Grübelneigung haben, sondern auch dazu neigen, über die Schlafprobleme zu grübeln, und insofern metakognitive Prozesse wirken, die auch bei Insomniepatienten auftreten. Interessant ist der Befund, 
dass die Diagnose einer Alptraumstörung ebenfalls mit vermehrten schlafbezogenen Metakognitionen einhergeht. Zum einen hängt das wahrscheinlich damit zusammen, dass diese Patientengruppe auch eine verringerte subjektive Schlafqualität angibt [10], aber möglicherweise auch mit dem Nachdenken über die Ursachen von Alpträumen. Schredl et al. [13] konnten zeigen, dass Personen, die darüber nachdenken, ob die Alpträume schlimme Dinge in der Zukunft vorhersagen und/oder auf unbewusste Ängste zurückzuführen sind, mehr unter Alpträumen leiden. Das heißt, dass auch der Umgang mit alptraumbezogenen Gedanken - im Sinne der metakognitiven Theorie - im Rahmen der Alptraumstörung klinisch relevant ist.

Insgesamt zeigte sich, dass die deutsche 20-Item-Version des MCQ-I schlafbezogene Metakognitionen reliabel und valide erfassen kann. Aufgrund der engen Verzahnung zwischen schlafbezogenen Kognitionen und schlafbezogenen Metakognitionen wäre eine Behandlungsstudie interessant, die die klassische kognitive Verhaltenstherapie der Insomnie (CBT-I) [5] mit einer reinen metakognitiven Therapie (MCT) [16] vergleicht. Wenn den Metakognitionen eine bedeutende Rolle in der Insomnie-Ätiologie zukommen sollte, wäre eine deutliche Besserung durch eine nicht-schlafspezifische MCT zu erwarten.

\section{Korrespondenzadresse}

apl. Prof. Michael Schredl

Schlaflabor, Zentralinstitut für Seelische Gesundheit, Medizinische Fakultät Mannheim, Universität Heidelberg

Postfach 1221 20, 68072 Mannheim,

Deutschland

Michael.Schredl@zi-mannheim.de

Funding. Open Access funding enabled and organized by Projekt DEAL.

\section{Einhaltung ethischer Richtlinien}

Interessenkonflikt. M. Schredl, M. Schackert, G.B. Feld und C. Schilling geben an, dass kein Interessenkonflikt besteht.

Für diesen Beitrag wurden von den Autoren keine Studien an Menschen oder Tieren durchgeführt. Für die aufgeführten Studien gelten die jeweils dort angege- benen ethischen Richtlinien. Die Ethikkommission II der Medizinischen Fakultät Mannheim/Universität Heidelberg hatte nach eingehender Prüfung keine ethischen und/oder berufsrechtlichen Einwände gegen die Auswertung der klinischen Daten.

Open Access. Dieser Artikel wird unter der Creative Commons Namensnennung 4.0 International Lizenz veröffentlicht, welche die Nutzung, Vervielfältigung, Bearbeitung, Verbreitung und Wiedergabe in jeglichem Medium und Format erlaubt, sofern Sie den/die ursprünglichen Autor(en) und die Quelle ordnungsgemäß nennen, einen Link zur Creative Commons Lizenz beifügen und angeben, ob Änderungen vorgenommen wurden.

Die in diesem Artikel enthaltenen Bilder und sonstiges Drittmaterial unterliegen ebenfalls der genannten Creative Commons Lizenz, sofern sich aus der Abbildungslegende nichts anderes ergibt. Sofern das betreffende Material nicht unter der genannten Creative Commons Lizenz steht und die betreffende Handlung nicht nach gesetzlichen Vorschriften erlaubt ist, ist für die oben aufgeführten Weiterverwendungen des Materials die Einwilligung des jeweiligen Rechteinhabers einzuholen.

Weitere Details zur Lizenz entnehmen Sie bitte der Lizenzinformation auf http://creativecommons.org/ licenses/by/4.0/deed.de.

\section{Literatur}

1. Amara AW, Maddox MH (2017) Epidemiology of sleep medicine. In: Kryger M, Roth T, Dement WC (Hrsg) Principles and practice of sleep medicine, 6. Aufl. Elsevier, Philidelphia, S627-637

2. American Academy of Sleep Medicine (2007) The AASM manual for the scoring of sleep and associated events: rules, terminology, and technical specifications. American Academy of Sleep Medicine, Westchester

3. American Academy of Sleep Medicine (2014) The international classification of sleep disorders. (ICSD-3). AASM, Darien

4. Feng G, Han M, Li X et al (2020) The clinical effectiveness of cognitive behavioral therapy for patients with insomnia and depression: a systematic review and meta-analysis. Evid Based Complement Alternat Med. https://doi.org/10. 1155/2020/8071821

5. Müller T, Paterok B (2010) Schlaftraining: Ein Therapiemanual zur Behandlung von Schlafstörungen, 2. Aufl. Hogrefe, Göttingen

6. Ong JC, UlmerCS, Manber R(2012) Improving sleep with mindfulness and acceptance:a metacognitive model of insomnia. Behav Res Ther 50:651-660

7. Palagini L, Mauri M, Dell'osso Letal (2016) Trait-and pre-sleep-state-dependent arousal in insomnia disorders: what role may sleep reactivity and sleeprelated metacognitions play? A pilot study. Sleep Med 25:42-48

8. PalaginiL, Ong JC, RiemannD(2017)Themediating role of sleep-related metacognitive processes in trait and pre-sleep state hyperarousal in insomnia disorder.J Psychosom Res 99:59-65

9. Palagini L, Piarulli A, Menicucci D et al (2014) Metacognitive beliefs relate specifically to sleep quality in primary insomnia: a pilot study. Sleep Med 15:918-922

10. Paul F, Schredl M, Alpers GW (2015) Nightmares affect the experience of sleep quality but not sleep architecture: an ambulatory polysomnographic study. Bord Personal Disord Emot Dysregul 2:1-9

11. Perlis ML, Ellis JG, Demichele Kloss J et al (2017) Etiology and pathophysiology of insomnia. In: Kryger M, Roth T, Dement WC (Hrsg) Principles and practice of sleep medicine, 6 . Aufl. Elsevier Philadelphia, S769-784

12. Rost J(1996) Lehrbuch Testtheorie. Huber, Bern

13. Schredl M, Holyba L, Köllmer T et al (2019) Nightmare distress, nightmare frequency, and beliefs about nightmares. Int JDream Res 12:60-66

14. Stuck BA, Maurer JT, Schlarb AA et al (2018) Praxis der Schlafmedizin. Springer, Heidelberg

15. Waine J, Broomfield NM, Banham S et al (2009) Metacognitive beliefs in primary insomnia: developing and validating the Metacognitions Questionnaire-Insomnia (MCQ-I). J Behav Ther Exp Psychiatry 40:15-23

16. Wells A (2011) Metakognitive Therapie be Angststörungen und Depression. Beltz, Weinheim

17. Wells A, Cartwright-Hatton S (2004) A short form of the metacognitions questionnaire: properties of the MCQ-30. Behav Res Ther 42:385-396

18. Zhang B, Wing YK (2006) Sex differences in insomnia:a meta-analysis. Sleep 29:85-93 